\title{
Elective Sonolucent Cranioplasty for Real-Time Ultrasound Monitoring of Flow and Patency of an Extra- to Intracranial Bypass
}

\author{
Caroline Hadley, MD, Robert North, MD, Visish Srinivasan, MD, Peter Kan, MD, \\ and Jan-Karl Burkhardt, MD
}

\begin{abstract}
Cross-sectional imaging studies or catheter angiogram are the imaging modalities of choice to evaluate bypass patency after extra- to intracranial (EC-IC) bypass surgery. Although providing accurate results, these imaging modalities are time-consuming and/or present radiation risk for the patient. Ultrasound imaging is a fast and widely available imaging modality, but is limited in this setting due to the non-sonolucent autologous bone flap covering the bypass after surgery. The recently FDA approved clear polymethyl methacrylate (PMMA) cranioplasty implant overcomes this limitation by its sonolucent characteristic, but has not yet been used in the setting of EC-IC bypass surgery. Here, the authors describe for the first time the feasibility of an elective sonolucent cranioplasty to monitor flow and patency of an EC-IC bypass in real time using ultrasound. This moyamoya patient underwent a direct superficial temporal artery to middle cerebral artery (STA-MCA) bypass, after which a PMMA implant was used to close the craniotomy defect, instead of reimplanting the autologous bone flap. Immediate postoperative bedside transcranioplasty ultrasound confirmed bypass patency and allowed for quantitative flow measurements as well as for exclusion of postoperative hemorrhage. Postoperative CTA and catheter angiogram confirmed patency of the bypass without complications. This report shows for the first time that this technique is feasible and permits bedside transcranioplasty ultrasound assessment of bypass flow in real time, confirmed with angiography. This technique may permit easy comparison of baseline findings with follow up assessments and facilitate less invasive monitoring of bypass patency.
\end{abstract}

Key Words: Extra- to intracranial bypass, real-time ultrasound monitoring, sonolucent cranioplasty

(J Craniofac Surg 2020;31: 622-624)

From the Department of Neurosurgery, Baylor College of Medicine, Houston, TX.

Received November 12, 2019.

Accepted for publication November 17, 2019.

Address correspondence and reprint requests to Jan-Karl Burkhardt, MD, Department of Neurosurgery, Baylor College of Medicine Medical Center, 7200 Cambridge Street, Suite 9B, Houston, TX, 77030; E-Mail: Jan-Karl.Burkhardt@bcm.edu

The authors have no conflicts of interest to disclose.

This is an open access article distributed under the terms of the Creative Commons Attribution-Non Commercial-No Derivatives License 4.0 (CCBY-NC-ND), where it is permissible to download and share the work provided it is properly cited. The work cannot be changed in any way or used commercially without permission from the journal.

Copyright (c) 2020 The Author(s). Published by Wolters Kluwer Health, Inc. on behalf of Mutaz B. Habal, MD.

ISSN: $1049-2275$

DOI: $10.1097 /$ SCS.0000000000006225
E xtra- to intracranial (EC-IC) bypass surgery is the treatment of E choice for patients with Moyamoya disease (MMD), a progressive disease of the intracranial vasculature, eventually leading to occlusion of the bilateral internal carotid arteries with frequent ischemic and/or hemorrhagic stroke complications. ${ }^{1}$ The most common EC-IC bypass type is the direct superficial temporal artery to middle cerebral artery (STA-MCA) bypass. ${ }^{2}$ To assess bypass patency after surgery or change of blood flow across the bypass over time cross-sectional imaging such as computed-tomography angiography (CTA) or magnetic resonance angiography (MRA) or invasive imaging such as a formal angiogram are required. Although highly accurate in evaluating bypass patency, these imaging modalities are time-consuming, not feasible at the bedside, and may present a radiation risk for the patient, especially in a patient population requiring multiple follow-up scans for the disease in the future. Eliminating additional radiation exposure and the need for an invasive procedure in favor of non-invasive, bedside, real time imaging would be beneficial to the patient.

As previously described, ultrasonography is an ideal imaging modality for frequent, real-time follow-up, as the image acquisition time is fast, it is widely available, it is non-invasive, and it requires no radiation exposure. ${ }^{3,4}$ With conventional craniotomy, ultrasound is not feasible due to the autologous bone flap covering most of the donor artery and bypass anastomosis site after surgery., ${ }^{3,4}$ The recently FDA approved polymethyl methacrylate (PMMA) cranioplasty implant (Longeviti Neuro Solutions, Hunt Valley, MD) is both transparent and sonolucent and may overcome this limitation, but has thus far not been used in the setting of EC-IC bypass surgery. Here, we describe for the first time the feasibility of using a sonolucent cranioplasty in the setting of EC-IC bypass surgery to monitor blood flow and patency of the EC-IC bypass in real-time postoperatively using ultrasound.

\section{MATERIALS AND METHODS}

Both the bypass procedure as well as the use of the PMMA cranioplasty implant, as opposed to the autologous bone flap, was discussed with the patient prior to surgery and written consent obtained. Standard direct STA-MCA bypass technique was performed using 10-0 nylon sutures (Ethilon, BV 75-3). Following successful anastomosis, the craniotomy defect was closed with a PMMA cranioplasty implant (Longeviti Neuro Solutions), which was drilled to fit the defect. The implant was affixed to the skull using the Stryker cranial plating system (Stryker, Kalamazoo, MI).

\section{RESULTS}

This 37-year-old woman with symptomatic MMD and previous right-sided direct STA-MCA bypass underwent a left-sided direct STA-MCA bypass for repeated TIAs. Standard direct STA-MCA bypass technique included microsurgical harvest of the donor STA, small craniotomy $(3 \times 3 \mathrm{~cm})$ and cruciate dural opening. A recipient MCA branch close to the sylvian fissure (M3-M4 segment) was 


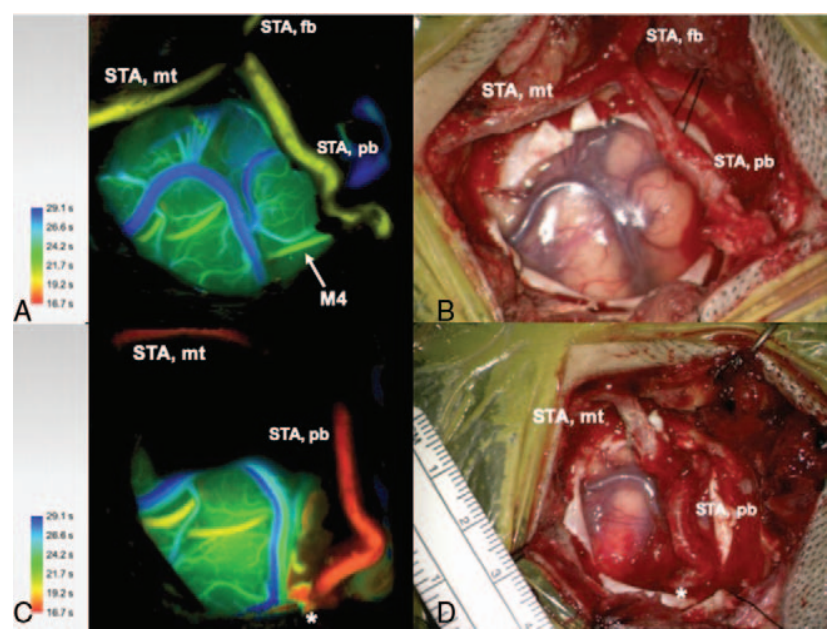

FIGURE 1. Indocyanine green (ICG) angiography color coded flow 800 (A) and microscopic image (B) before STA-MCA bypass after harvest of the STA, craniotomy and dura opening. The marked M4 MCA branch was selected for the bypass as recipient. STA, superficial temporal artery, STA mt, STA main trunk, STA fb, STA frontal branch, STA pb, STA parietal branch. ICG angiography color coded flow 800 (C) and microscopic image (D) after STA-MCA bypass with patent anastomosis and increased flow compared to pre-bypass.

chosen based on indocyanine green (ICG) angiography with the largest diameter and most delayed flow compared to the STA. After fish-mouthing of the parietal branch of the STA and cross clamping of the selected M3-4 vessel segment, the MCA vessel segment was opened and two 10-0 nylon anchoring sutures (Ethilon BV 75-3) were placed. The anastomosis was completed using an end (STA)to-side (MCA) continuous suture technique.

Initial patency of the bypass was confirmed using ICG angiography and with a microdoppler probe (Fig. 1). The bone flap was used to create a template and the PMMA implant was shaped to match the craniotomy defect and fixed over the craniotomy defect using titanium screws, taking care to allow sufficient space inferiorly to ensure no compression of the STA graft as it coursed intracranially (Fig. 2).

After skin closure, postoperative bedside transcranioplasty ultrasound confirmed bypass patency in real-time and allowed for

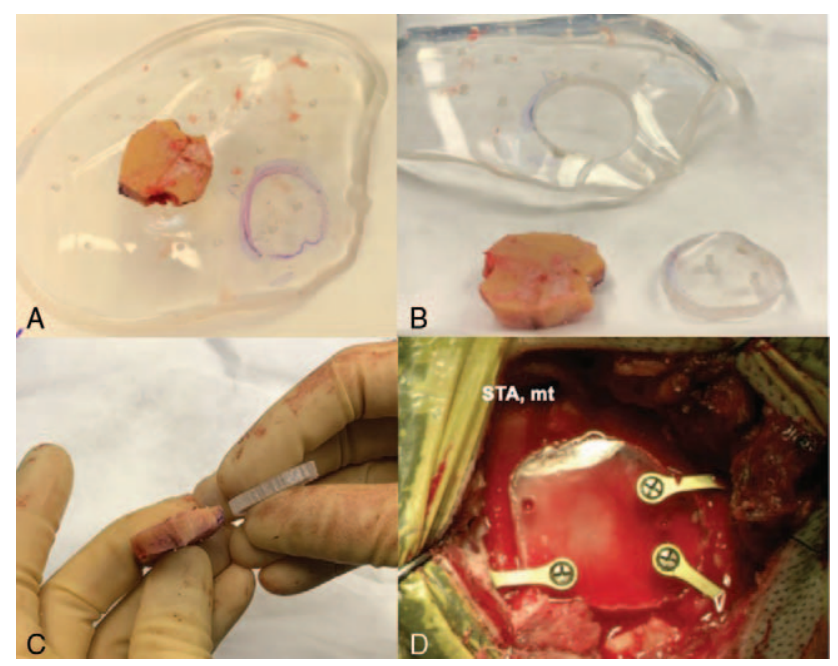

FIGURE 2. The bone flap was used to determine the size and shape of the clear PMMA implant (A) and an approximately $3 \times 3 \mathrm{~cm}$ cranioplasty implant was cut out $(B, C)$ and fixated over the craniotomy defect using titanium screws and mini-plates allowing enough space inferiorly without compression of the STA graft moving from extra- to intracranial. STA mt, superficial temporal artery main trunk.

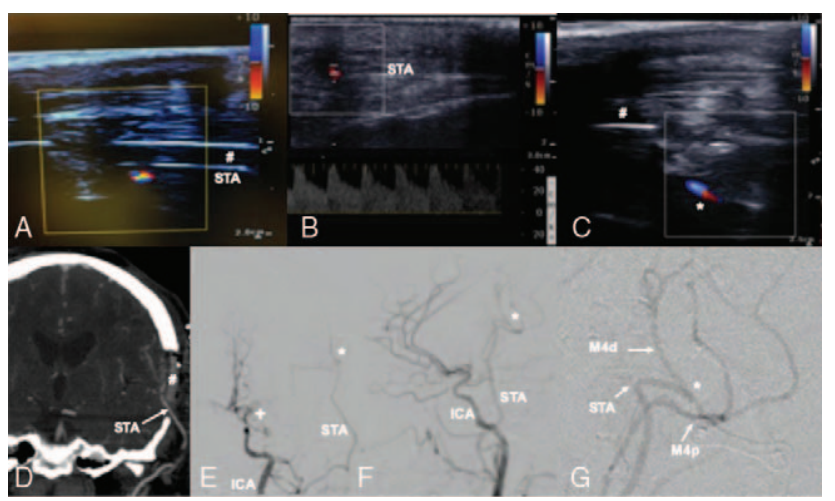

FIGURE 3. Transcranioplasty ultrasound confirmed flow in donor STA under the cranioplasty (\#) in real-time (A), allowed for quantitative flow measurements of the proximal STA donor vessel (B) and confirmed patency of the anastomosis (C). Postoperative CTA coronal view (D) and catheter angiogram in PA (E) lateral (F-G) planes confirmed bypass patency. * bypass anastomosis, + moyamoya disease vessel occlusion. \# transparent PMMA cranioplasty implant.

quantitative flow measurements (Fig. 3). The PMMA implant was identifiable on ultrasound and the STA graft, as well as distal MCA vessels on the cortical surface could be clearly visualized beneath the cranioplasty using doppler (Fig. 3). The brain parenchyma was also well visualized, without evidence of post-op hematoma. These findings were confirmed using postoperative CTA and formal cerebral angiogram (Fig. 3). The patient recovered well from surgery without any complications or new neurological deficits and was appropriate for discharge on postop day 5 .

\section{DISCUSSION}

The PMMA cranioplasty is a newly FDA approved cranioplasty implant that is both transparent and sonolucent. This far, it has been mainly used for patients with craniectomy defects. ${ }^{5}$ It has been previously demonstrated that high quality ultrasound images, comparable to cross-sectional imaging modalities, can be acquired across this implant. ${ }^{6}$ This has been used previously to monitor ventricular size and evaluate for complications, such as epidural or subdural hematomas.

Applying this technique to bypass surgery may be beneficial, as monitoring flow through the bypass within the first days after surgery is important in avoiding early occlusion. ${ }^{7}$ Additionally, when compared to other imaging modalities, ultrasound can be used at bedside without radiation exposure, contrast administration, or need for an invasive procedure. This is the first report electively using a sonolucent cranioplasty implant to allow for real time monitoring of flow through an EC-IC bypass. Postoperatively, were able to qualitatively monitor the whole length of the donor STA to the anastomosis site and were also able to quantitatively measure the wave form of the flow, while also assessing for postoperative hematoma formation. This provides valuable information about vessel caliber and flow through the bypass immediately post-op, which can be used to assess for vasospasm or occlusion in the postop period, and for comparison during long-term follow-up.

In the future, use of a PMMA implant may also permit visualization of developing collateral vessels over time, which could provide information about the timeline of vascular collateralization. Beyond vascular imaging, PMMA cranioplasty may be also be cosmetically beneficial to bypass patients, as the small bone flaps typically made during bypasses are prone to resorption. Given this risk, an elective cranioplasty may offer a better cosmetic outcome for the patient. ${ }^{8}$ Determination of this effect will of course require further evaluation with longer follow-up of a larger cohort of patients. 
The PMMA cranioplasty implants may represent a useful adjunct to EC-IC bypass surgery by facilitating real-time monitoring of bypass patency using transcranioplasty ultrasound. Further evaluation in additional patients will be necessary to truly assess the reliability of vessel imaging, utility of non-invasive vascular imaging, and potential cosmetic benefit.

\section{REFERENCES}

1. Baaj AA, Agazzi S, Sayed ZA, et al. Surgical management of moyamoya disease: a review. Neurosurg Focus 2009;26:E7

2. Burkhardt JK, Lawton MT. Practice trends in intracranial bypass surgery in a 21-year experience. World Neurosurg 2019;125:e717-e722

3. Morton RP, Abecassis IJ, Moore AE, et al. The use of ultrasound for postoperative monitoring of cerebral bypass grafts: a technical report. J Clin Neurosci 2017;40:169-174
4. Morton RP, Moore AE, Barber J, et al. Monitoring flow in extracranialintracranial bypass grafts using duplex ultrasonography: a single-center experience in 80 grafts over 8 years. Neurosurgery 2014;74:62-70

5. Belzberg M, Shalom NB, Lu A, et al. Transcranioplasty ultrasound through a sonolucent cranial implant made of polymethyl methacrylate: phantom study comparing ultrasound, computed tomography, and magnetic resonance imaging. J Craniofac Surg 2019;30:e626-e629

6. Belzberg M, Shalom NB, Yuhanna E, et al. Sonolucent cranial implants: cadaveric study and clinical findings supporting diagnostic and therapeutic transcranioplasty ultrasound. J Craniofac Surg 2019;30:1456-1461

7. Yoon S, Burkhardt JK, Lawton MT. Long-term patency in cerebral revascularization surgery: an analysis of a consecutive series of 430 bypasses. J Neurosurg 2018:1-8

8. Fischer CM, Burkhardt JK, Sarnthein J, et al. Aesthetic outcome in patients after polymethyl-methacrylate (PMMA) cranioplasty - a questionnaire-based single-centre study. Neurol Res 2012;34:281-285

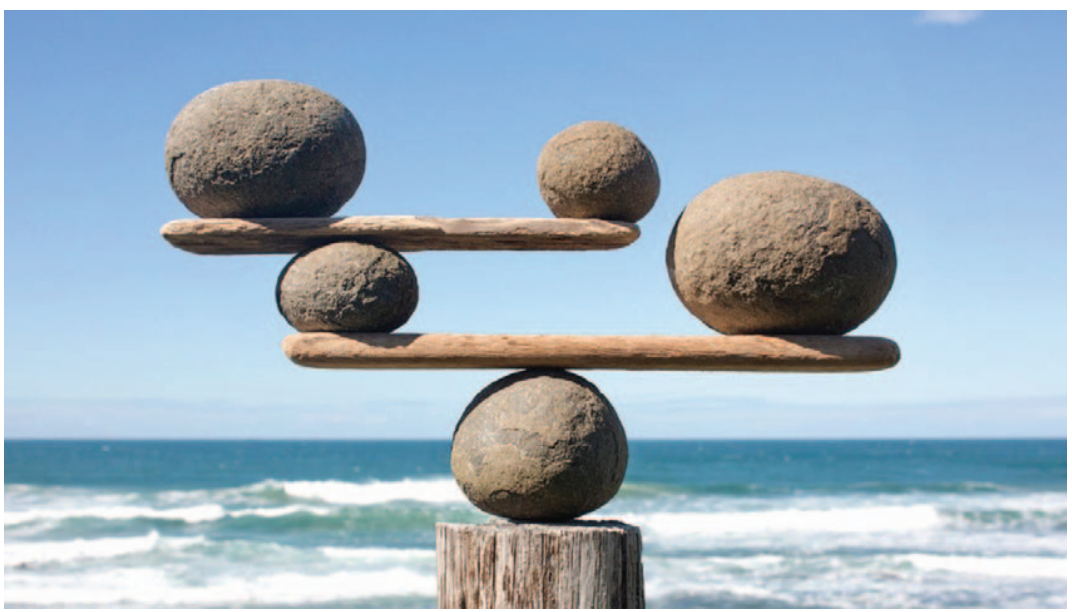

Balance. 